\title{
Research Paper \\ Introducing a Formula for Obtaining the Total Deviation Index in Aging Studies
}

\author{
Andisheh Bakhshi' ${ }^{1}$ (1), *Enayatollah Bakhshi² (1)
}

1. School of Computing, Engineering and Physical Sciences, Paisley Campus, University of the West of Scotland, Glasgow. 2. Department of Biostatistics, University of Social Welfare and Rehabilitation Sciences, Tehran, Iran.

dteation: Bakhshi A, Bakhshi E. [Application of Total Deviation Index in Aging Studies (Persian)]. Iranian Journal of Ageing. 2020; 15(3):278-285. https://doi.org/10.32598/sija.15.3.827.2

doi https://doi.org/10.32598/sija.15.3.827.2

Key words: Intraclass correlation coefficient, Total Deviation Index, Quality of Life, Elderly

\section{ABSTRACT}

Objectives Since the study on the elderly people and recording related data requires more accuracy, the calculation of measurement error is very important. This study aims to introduce a simple formula to obtain and interpret the Total Deviation Index (TDI) and encourage researchers to use it as a suitable too to determine the reliability in psychometrics studies.

Methods \& Materials In this cross-sectional study conducted in 2019, participants were 105 older adults living in Tehran, Iran. The 36-Item Short Form Health Survey (SF-36) questionnaire was completed by them within two weeks. The Intraclass Correlation Coefficient (ICC) and TDI values were calculated and compared for each subscale of the SF-36.

Results The lowest TDI value belonged to the "physical functioning" subscale, while the "emotional problems" subscale had the highest TDI value. At a 95\% confidence interval, the maximum measurement errors in physical functioning and emotional problems were reported 22 and 34 (out of 100), respectively. Conclusion Since the ICC cannot determine how much of the difference in the subscale scores is related to the measurement error, and given the simple interpretation of the TDI, it is recommended that researchers use the proposed formula and obtain the TDI value for the reliability in psychometrics studies.

\section{Extended Abstract}

\section{Introduction}

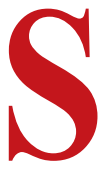

ince the study on the elderly people and recording related data requires more accuracy, the calculation of measurement error is very important. Each questionnaire must have good validity and reliability in order to be able to collect acceptable data. Although many studies use Intraclass Correlation Coefficient (ICC) (or Cronbach's alpha) as the reliability coefficient of the questionnaire, this indicator cannot determine how much of the difference in the subscales is related to measurement error. This study aims to introduce a simple formula to calculate the Total Deviation Index (TDI), interpret it and encourage researchers to use this index as a suitable tool to determine the reliability of psychometrics studies and its application in the Quality of Life questionnaire.

\section{Methods \& Materials}

In this cross-sectional study, 105 elderly people living in Tehran, Iran were selected using a convenience sampling technique in 2019. In order to determine their quality of life, 36-Item Short Form Health Survey (SF-36) questionnaire was completed in a period of two weeks and the score of each person (ranged 0-100) was calculated for the whole

\section{* Corresponding Author:}

Enayatollah Bakhshi, PhD.

Address: Department of Biostatistics, University of Social Welfare and Rehabilitation Sciences, Tehran, Iran. Tel: +98 (21) 22180146

E-mail: bakhshi@razi.tums.ac.ir 
questionnaire as well as for its 8 sub-scales. Then, the ICC and TDI values for the subscales of the questionnaire were calculated and compared.

\section{Results}

The Results showed that the lowest value of the TDI was related to the subscale "physical functioning" and the highest value was related to the subscale "emotional problems". At a 95\% confidence interval, the maximum measurement error under the physical functioning and emotional problems subscales was 22 and 34 (out of 100), respectively. For the sub-scales of physical health problems, bodily pain, general health, vitality, social functioning, and mental health, the maximum measurement error values were $31.59,24.54,33.69,29.32,78.33$ and 24.43 , respectively (out of 100). According to Pearson correlation test Results, the TDI values were not significantly correlated with the ICC values. That is, if the ICC value is high, the TDI value is not necessary to be low, and vice versa. Although a scale from 0 to 100 was used for scoring the SF-36 subscales, the TDI can be obtained and interpreted similarly in any case where any other scoring methods are used. Moreover, although the TDI values in our study were interpreted at a 95\% confidence interval (due to its popularity), it can also be interpreted at any other probability rates.

\section{Conclusion}

Since the ICC cannot determine how much of the difference in the subscale scores is related to the measurement error, and given the simple interpretation of the TDI, it is recommended that researchers report the TDI value using the proposed formula for the reliability in psychometrics studies.

\section{Ethical Considerations}

\section{Compliance with ethical guidelines}

This study was approved by the Ethics Committee of University of Social Welfare and Rehabilitation Sciences (Code: IR.USWR.REC.13960379).

\section{Funding}

This research did not receive any specific grant from funding agencies in the public, commercial, or not-forprofit sectors.

\section{Authors' contributions}

Both authors contributed equally in preparing all parts of the research.

\section{Conflicts of interest}

The authors declared no conflict of interest. 
This Page Intentionally Left Blank 


\section{كاربرد شاخص انحراف كل در مطالعات سالمندى}

انديشه بخشى' (1) 'منايتاله بخشى

ا. دانشكده محاسباته مهندسى و علوم فيزيك، دانشّكاه غرب اسكاثلند، كلاسكّو.

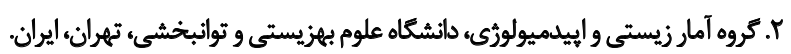

\section{حكיد}

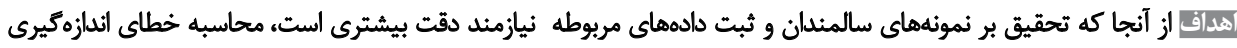

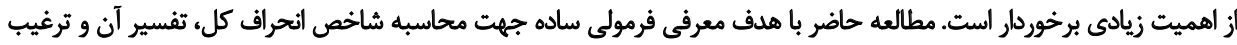

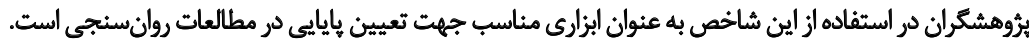

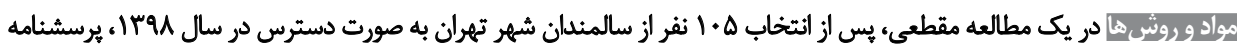

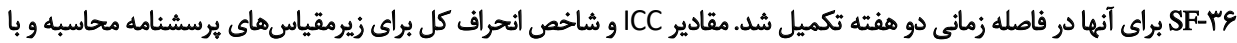

$$
\text { هم مقايسه شدند. }
$$

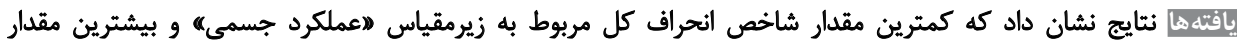

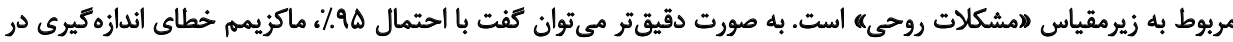

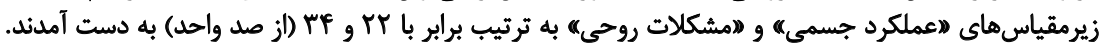

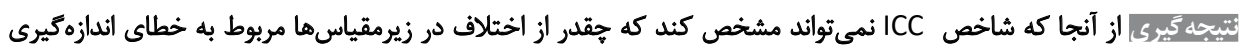

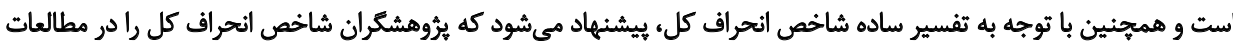
بايايى كزارش نمايند.
تاريخ دريافت: 19 مرداد

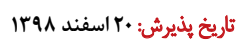

تاريخ انتشار: 11 تير 149
كليدوأرهاها:

ضريب همبستئى درون

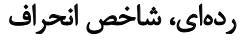

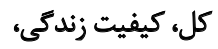
سالمند

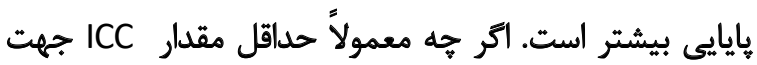

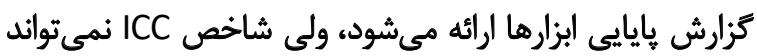

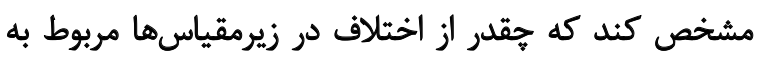

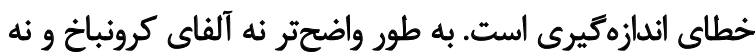

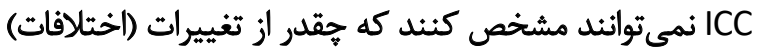

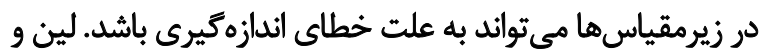

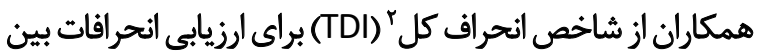

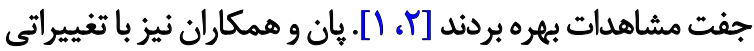
در اين شاخص، از آن جهت محاسبه خطائ اندازئ بازيكيرى استفاده

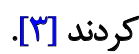

اندازمكيرى "اكيفيت زندكى بَه به عنوان يكى از شاخصهاى

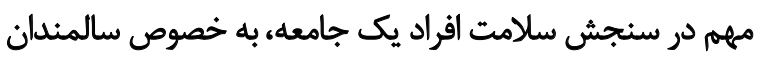

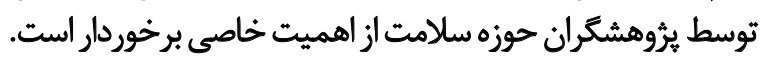

2. Total Deviation Index

3. Quality of Life

1. Intraclass Correlation Coefficient

dolis

از آنجا كه تحقيق بر نمونههاى سالمندان و ثبت دادههاي

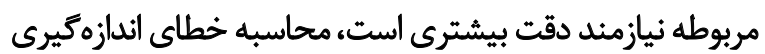

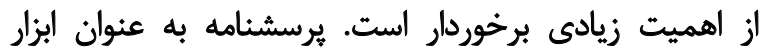

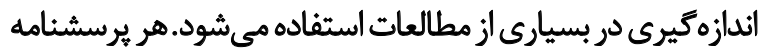

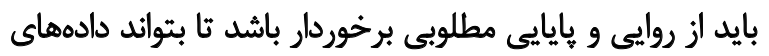

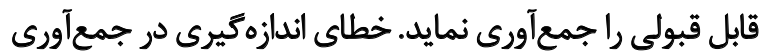

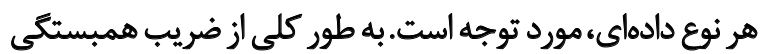

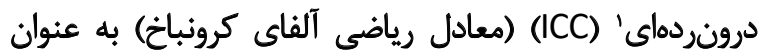
ضريب يايايى يرسشنامه استفاده مىشود.

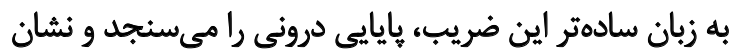

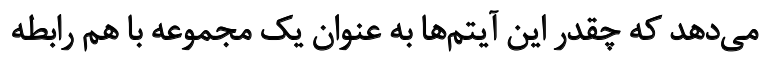

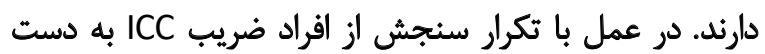

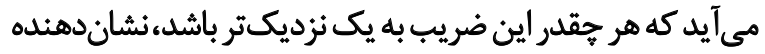

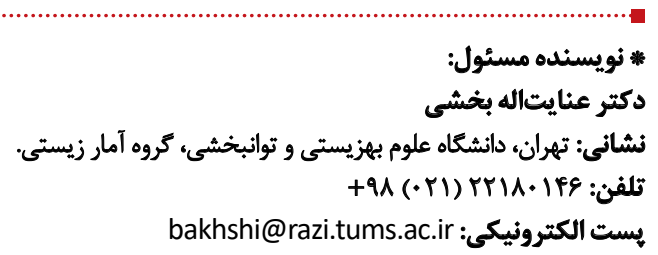




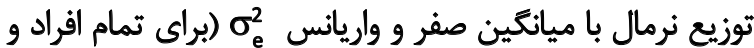

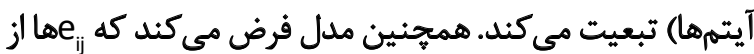

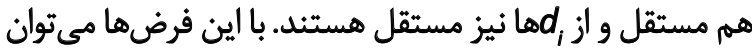

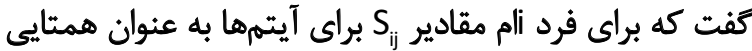

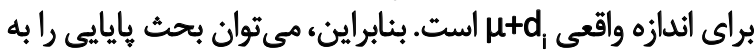

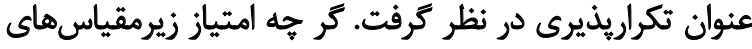

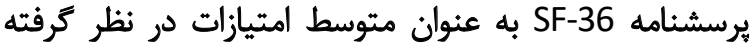

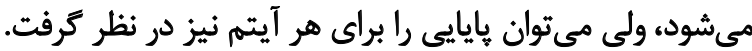

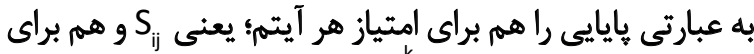

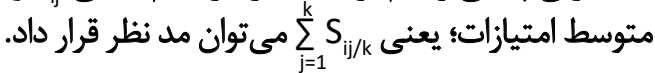

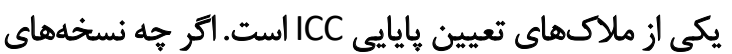

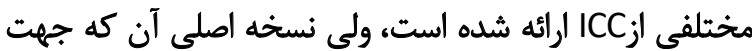

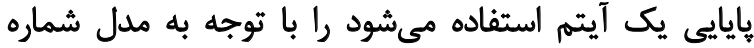
(1) مىتوان به صورت نسبت واريانس مقدار واقعى به واريانس مشاهده شده به صورت زير توسط فرمول شماره (T) تعريف كرد:

2.

$$
\begin{aligned}
& \operatorname{IcC} c_{1}=\frac{\operatorname{Var}\left(\mu+d_{i}\right)}{\operatorname{Var}\left(S_{i j}\right)}=\frac{\operatorname{Var}\left(S_{i j}+e_{i j}\right)}{\operatorname{Var}\left(S_{i j}\right)}=1-\frac{\operatorname{Var}\left(e_{i j}\right)}{\operatorname{Var}\left(S_{i j}\right)} \\
& =1-\frac{\operatorname{Var}\left(e_{i j}\right)}{\operatorname{Var}\left(d_{i j}\right)+\operatorname{Var}\left(e_{i j}\right)}=\frac{\operatorname{Var}\left(d_{1}\right)}{\operatorname{Var}\left(d_{i}\right)+\operatorname{Var}\left(e_{i j}\right)}
\end{aligned}
$$

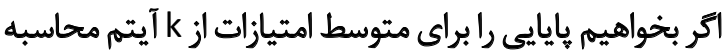

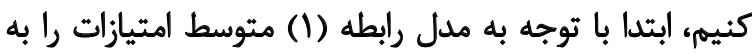
صورت زير محاسبه مى كنيم (فرمول شماره بآ): 3. $\frac{1}{k} \sum_{j=1}^{k} S_{i j}=\frac{1}{k} \sum_{j=1}^{k}\left(\mu+d_{i}+e_{i j}\right)=\mu+d_{l}+\frac{1}{k} \sum_{j=1}^{k}\left(e_{i j}\right)$ سيس ICC براي متوسط امتيازات حاصل از k آيتم به صورت زير توسط رابطه شماره (f) محاسبه مى شودي الرئ

4.

$I c c_{k}=\frac{\operatorname{Var}\left(e_{j}\right)}{\left[d_{i}+\frac{1}{k} \sum_{j=1}^{k}\left(e_{i j}\right)\right]}=\frac{\operatorname{Var}\left(e_{e}\right)}{\operatorname{Vard}{ }_{i} \frac{1}{k} \sum_{j=1}^{k}\left(e_{i j}\right)}=\frac{(k)\left(I c c_{i}\right)}{(k-1)\left(I c c_{i}\right)+1}$

واضح است كه با افزايش تعداد آيتمها بايايى افزايش مىيابد.

شاخص انحراف كل

اتر جهه استفاده از ICC در بسيارى از آزمونها رايج است، بايد

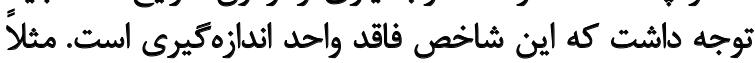

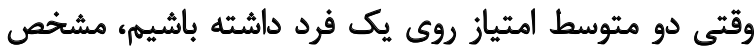

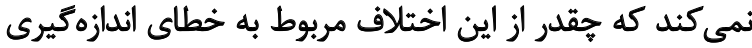

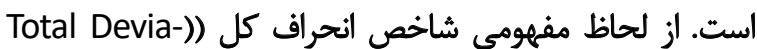

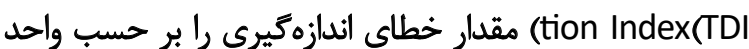

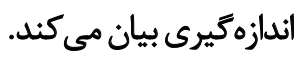

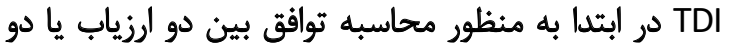

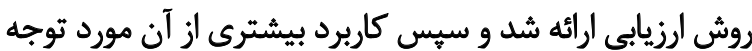

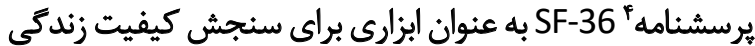

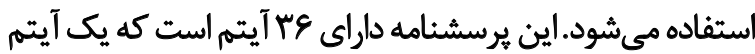

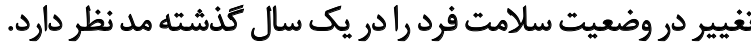

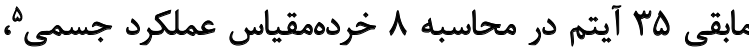

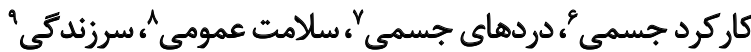

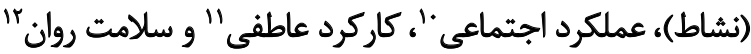

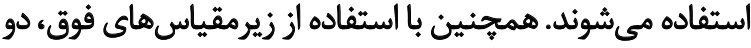

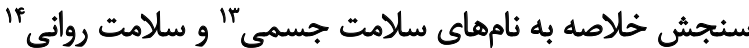

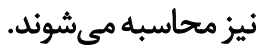

نمرات برسشنامه معمولاً به مقياس صفر تا صد تبديل شده

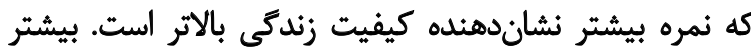

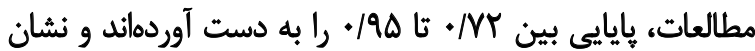

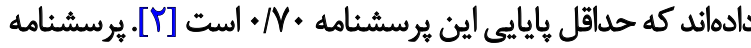

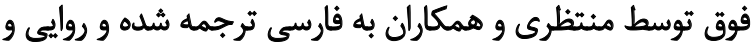

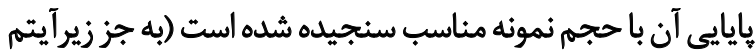

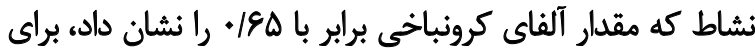

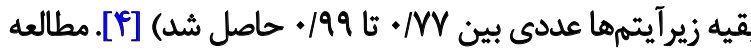

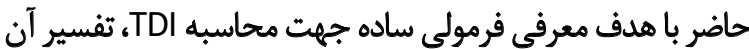

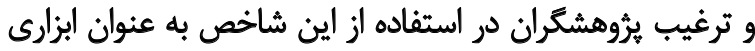

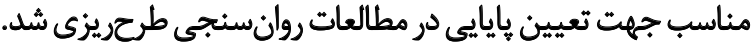

$$
\text { موش مطالعه }
$$

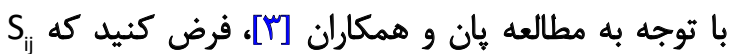

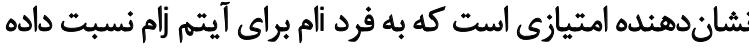

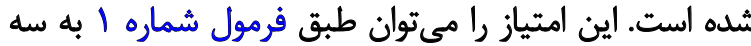
مؤلفه تقسيم كرد:

\section{1. $S_{i j}=\mu+d_{i}+e_{i j} \quad i=1,2 \ldots . . n, \quad j=1,2, \ldots . . n$}

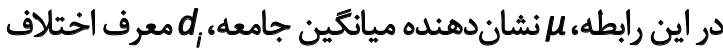

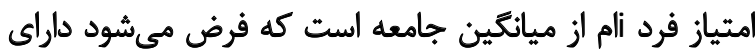

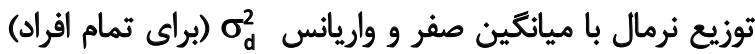

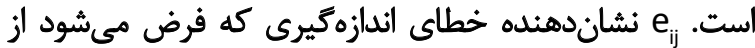

4. 36-Item Short Form Health Survey

5. Physical functioning

6. Role Limitation because of physical health problems

7. Bodily pain

8. General health perceptions

9. Vitality

10. Social functioning

11. Role limitations because of emotion of problems

12. Mental health

13. Physical Component Summary

14. Mental Component Summary 
جدول ا. محاسبه شاخص ائحراف كل براي برسشنامه وبr SF در نمونه هـ 1 ن نفرى از سالمندان

\begin{tabular}{|c|c|c|c|}
\hline TDI $(\% 95)^{*}$ & $\mathrm{ICC}$ & $\sigma$ & زيرمقياس \\
\hline$r r / l q$ & - /ar & PNHT & عملكرد جسمى \\
\hline$r v / \Delta q$ & $\cdot / M$ & $m / 91$ & مشكلات جسمى \\
\hline$T E / \Delta F$ &.$/ A V$ & $T H / A S$ & درد جسمى \\
\hline$m p / s q$ &.$M{ }^{c}$ & $M T / A F$ & سلامتت عمومى \\
\hline $\mathrm{ra/m}$ &.$/ N A$ & $M T / \Delta F$ & نشاط \\
\hline$\pi / N A$ & - AR & $r+/ p r$ & عملكرد اجتماعى \\
\hline$m e / m q$ & $\cdot / A r$ & Me/PT & مشكلات روحى \\
\hline$M$ & . IVA & $19 / 4 F$ & سلامت روان \\
\hline
\end{tabular}

و • درصد فرمول به صورت سادهتر در رابطهى شماره V قابل

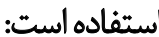

7. $\begin{cases}\operatorname{TDI}_{k}(95 \%)=\sigma_{\text {subscale Total }} & \sqrt{6.68\left(1-I C C_{k}\right)} \\ \operatorname{TDI}_{k}(90 \%)=\sigma_{\text {subscale Total }} & \sqrt{5.42\left(1-I C C_{k}\right)} \\ \operatorname{TDI}_{k}(80 \%)=\sigma_{\text {subscale Total }} & \sqrt{3.28\left(1-/ C C_{k}\right)}\end{cases}$

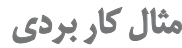

يس از انتخاب هـ أ سالمند از جامعه سالمندان در سطح

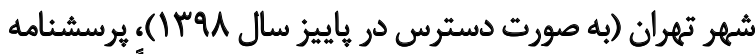

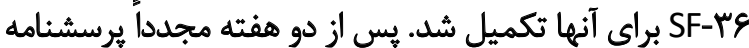

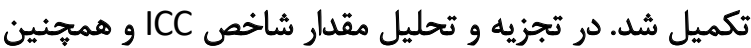

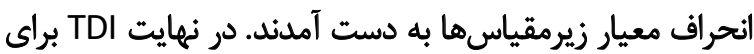

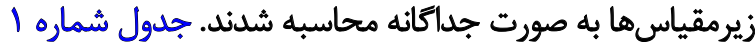

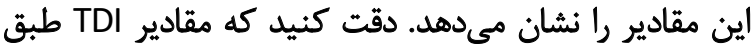

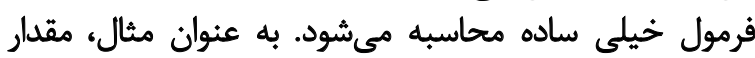

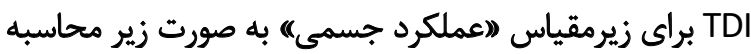

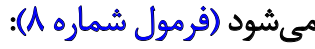

8. $T D I_{i}=\sigma_{\text {Total }} \sqrt{2 X_{(1,1-a)}^{2}(1-I C C)}$

$=28.32 \sqrt{2(3.84)(1-0.9)}=22.19$

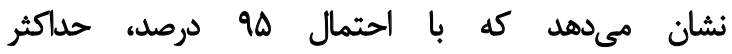

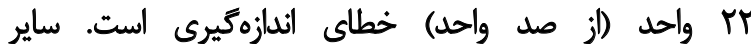

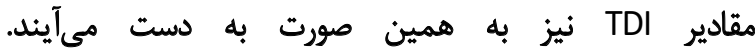

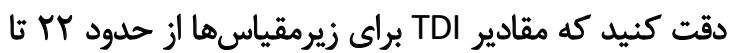
الح MF "اعملكرد جسمي) و بيشترين خطا مريوط به زئ زيرمقياس

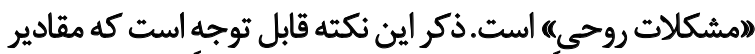

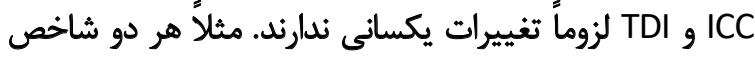

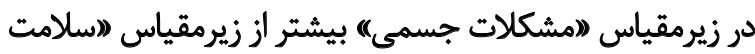

قرار كرفت. I, TDI شبيه ICC مي توان براى يك آيتهم و يا متوسط

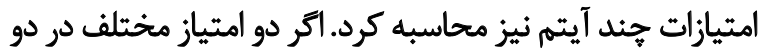

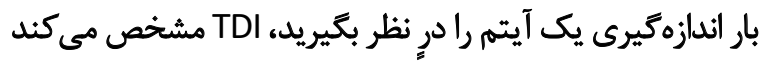

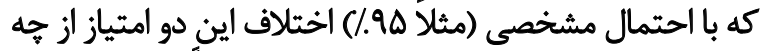

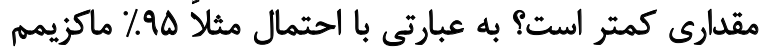

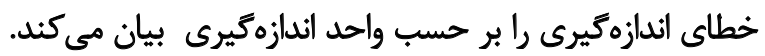

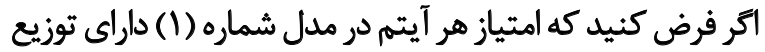
نرمال باشد، در اين صورت اختلاف هر دو امتياز نيز داراى توزيع

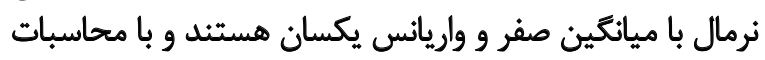
رياضى، مىثوان TDI براى يك آيتم راطبق روان رابطه شماره (ه) و و

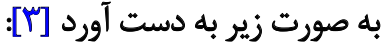

5. $T D I=\sigma_{\text {Total }} \sqrt{2 X_{(1,1-a)}^{2}\left(1-I C C_{1}\right)}$

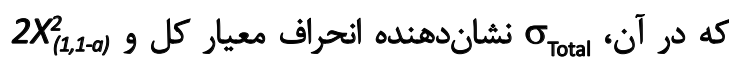
صدى توزيع كاسكور است كه از جدول مربوطه به دست مئ مئي آيد. دقت كنيد كه در بسيارى از هرسشنامهها، متوسط تعدادى از

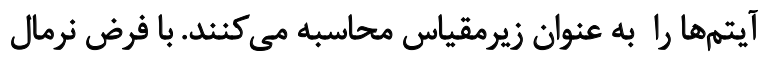

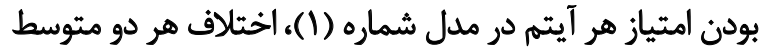

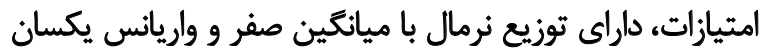

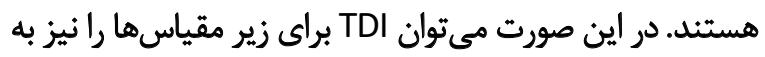

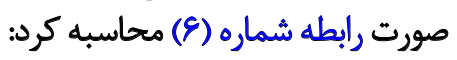

6. $T D I_{k}=\sigma_{\text {subscale Total }} \sqrt{2 X_{(1,1-a)}^{2}\left(1-I C C_{k}\right)}$

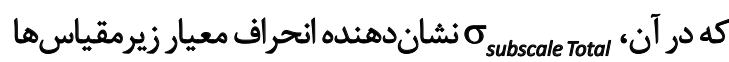

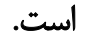

حالات خاص: دقت كنيد كه براى استفاده از فرمول شماره (ه)

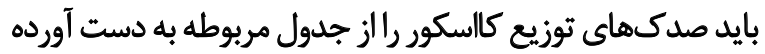

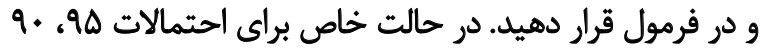




\section{ملاحظات اخلاقى

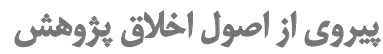

اين برؤهش بر كرفته از يك طرح تحقيقاتى است كه يرويوزال

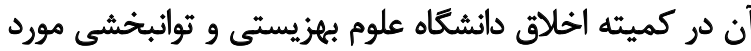
تأييد قرار كرفته است (كد: IR.USWR.REC.13960379).

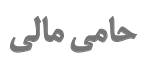

اين يرؤهش هيجكونه كمك مالى از سازمانىهاى دولتى، خصوصى وغيرانفاعى دريافت نكرده است.

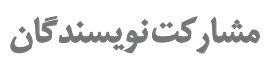

هر دو نويسنده در آمادمسازى اين مقاله مشاركت داشتهاند.

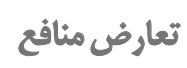

نويسندكان اظهار مى دارند كه اين يُؤوه هيج تعارض

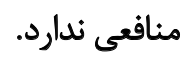

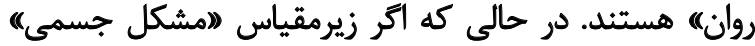

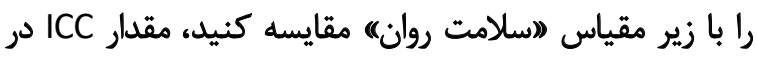

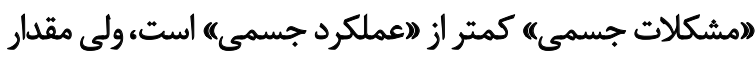

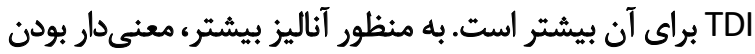

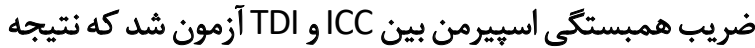
نشان داد آزمون همبستئى معنى دار نيست (P=0.551).

در اين مطالعه با استفاده از شاخصى به نام TDI حداكثر باكثر خطاى

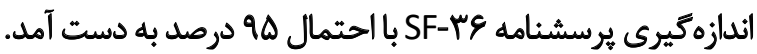

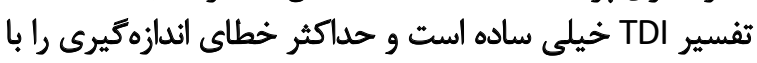

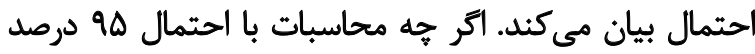

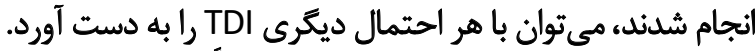

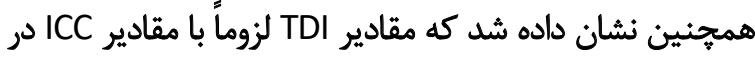

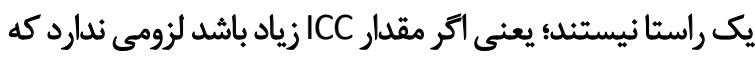

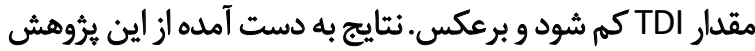

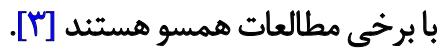

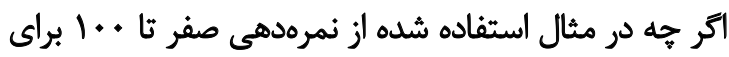

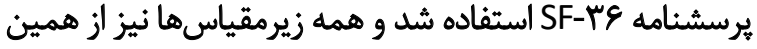

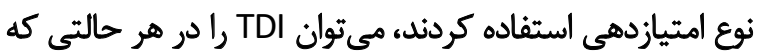

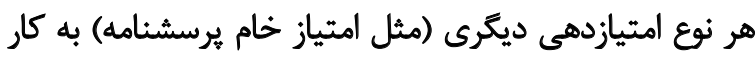

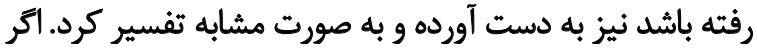

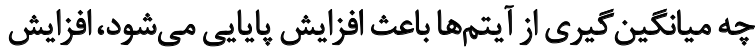
تعداد آيتمها نيز در افزايش هائيايى مؤثر است.

فرمول محاسبه TDI در اينجا با فرض نرمال بودن توزيع

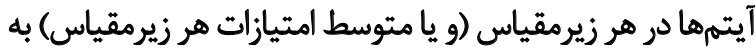

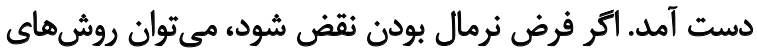

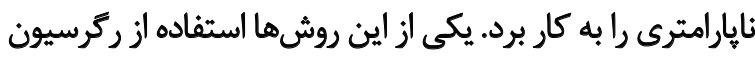

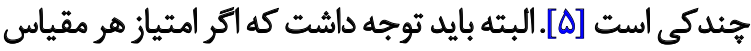

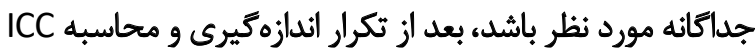

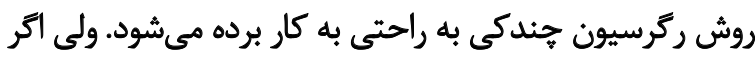

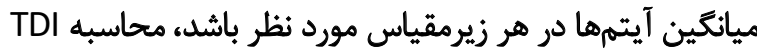

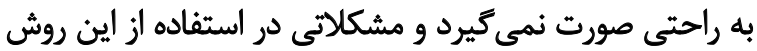

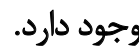

\section{نتيجلكيرى نهايى}

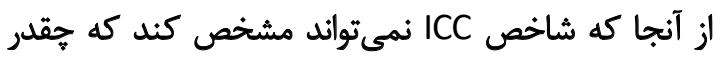

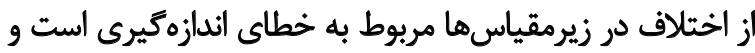

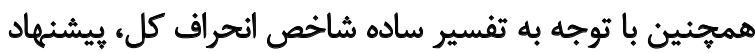

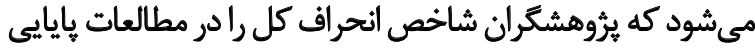

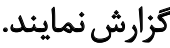




\section{References}

[1] Lin LI. Total deviation index for measuring individual agreement with applications in laboratory performance and bioequivalence. Statistics in Medicine. 2000; 19(2):255-70. [DOI:10.1002/ (sici)1097-0258(20000130)19:2<255::aid-sim293>3.0.co;2-8] [PMID]

[2] McHorney CA, Ware Jr JE, Rachel Lu JF, Sherbourne CD. The MOS 36-item Short-Form Health Survey (SF-36): III. Tests of data quality, scaling assumptions, and reliability across diverse patient groups. Medical Care. 1994; 32(1):40-66. [DOI:10.1097/00005650199401000-00004] [PMID]

[3] Pan Y, Barnhart HX. Methods for assessing the reliability of quality of life based on SF-36. Statistics in Medicine. 2016; 35(30):565665. [DOI:10.1002/sim.7085] [PMID]

[4] Montazeri A, Goshtasebi A, Vahdaninia M, Gandek B. The Short Form Health Survey (SF-36): Translation and validation study of the Iranian version. Quality of Life Research. 2005; 14(3):875-82. [DOI:10.1007/s11136-004-1014-5] [PMID]

[5] Lin L, Pan Y, Hedayat AS, Barnhart HX, Haber M. A simulation study of nonparametric total deviation index as a measure of agreement based on quantile regression. Journal of Biopharmaceutical Statistics. 2016; 26(5):937-50. [DOI:10.1080/10543406.20 15.1094812] [PMID] 